\title{
New-Onset Seizures in HIV Patients on Antiretroviral Therapy at a Tertiary Centre in South-West, Nigeria
}

\author{
Oshinaike Olajumoke ${ }^{1 *}$, Akinbami Akinsegun ${ }^{2}$, Okubadejo Njideka $^{3}$, Ojo Oluwadamilola ${ }^{3}$, \\ Ojelabi Olaitan ${ }^{1}$, Dosunmu Adedoyin ${ }^{2}$, Adediran Adewumi ${ }^{4}$, Dada Akinola ${ }^{1}$, Ajibola Sarah ${ }^{4}$ \\ ${ }^{1}$ Department of Medicine, Lagos State University College of Medicine, Lagos, Nigeria; ${ }^{2}$ Department of Haematology and Blood Trans- \\ fusion, Lagos State University College of Medicine, Lagos, Nigeria; ${ }^{3}$ Department of Medicine, College of Medicine, University of Lagos, \\ Lagos, Nigeria; ${ }^{4}$ Department of Haematology and Blood Transfusion, College of Medicine, University of Lagos, Lagos, Nigeria. \\ Email: *olajumoke68@yahoo.com
}

Received February $4^{\text {th }}, 2013$; revised March $5^{\text {th }}, 2013$; accepted March $14^{\text {th }}, 2013$

Copyright (C) 2013 Oshinaike Olajumoke et al. This is an open access article distributed under the Creative Commons Attribution License, which permits unrestricted use, distribution, and reproduction in any medium, provided the original work is properly cited.

\begin{abstract}
Background: Seizures are associated with neurological manifestations of HIV. They may be the presenting symptom and can occur at any disease stage. Aim: To determine the frequency and clinical aspects of new-onset seizures in patients with human immunodeficiency virus (HIV) infection. Methods: A study of an HIV-infected patient cohort on highly active anti-retroviral therapy (HAART) in the out-patients clinic of the Lagos state university teaching hospital, Nigeria. In a cross-sectional design, 308 HIV infected patients were recruited over a period of 1 year. Cases with a first seizure during this period were further examined. Details of demographic data, the first seizure date, seizure characteristics, neurologic complications and CD4 count at the time of the seizure were documented. Results: A total of 20 (6.5\%) had new-onset seizures during the study period. 6/20 (30\%) were males and 14/20 (70\%), females. Their ages ranged between $22-51$ years with a mean of $34.2 \pm 8.7$ years. The seizure was focal in $2 / 20(10 \%)$ of cases and generalised in $90 \%(18 / 20)$ of cases. A total of 13/20 (65\%) had recurrence of their seizures. None of the cases had focal neurological deficit at the time of the first seizure. The mean CD4 count was $165.3 \pm 145.7$. The mean duration on HAART was $19.5 \pm 12.7$ months. Cases with CD4 counts $\leq 200$ cells $/ \mathrm{mm}^{3}$ constituted $70 \%(14 / 20)$ whilst those with CD4 counts $>200$ made up 30\% $(6 / 20)[p=0.666]$. Conclusions: Seizures remain a significant neurological manifestation of HIV infection and has a high recurrence rate. It occurs more commonly in the advanced stage with severe immune suppression and may be attributable to HIV encephalopathy. Early treatment would reduce the burden and improve patient's quality of life.
\end{abstract}

Keywords: New-Onset Seizures; HIV/AIDS

\section{Introduction}

Seizures are frequent manifestations of central nervous system disorders in patients infected with human immunodeficiency virus (HIV). They are more common in advanced stages of the disease, although they may occur early in the course of illness with an estimated frequency of $11 \%$ to $17 \%$ and a recurrence rate as high as $70 \%$ [1]. They may be the presenting symptom in $18 \%$ of cases [2].

Approximately half of HIV-infected patients with seizures have no definite identifiable disease of the brain, and HIV encephalopathy seems to be the most likely cause of the seizures [3-5]. Seizures may also occur as a result of focal brain lesions such as opportunistic infec-

${ }^{*}$ Corresponding author. tions, (CNS toxoplasmosis, Progressive multifocal leukoencephalopathy and CNS lymphoma) neoplasms, and cerebrovascular diseases $[3,6,7]$. Others include cryptococcal abscesses, tuberculous abscesses, tuberculomas, nocardial abscesses, and syphilitic gummas [8-11]. Drug toxicity and metabolic derangement may act as precipitants [4]. Meningitis resulting from opportunistic infections is also a frequent source of seizures with cryptococcal meningitis being the most common $[4,12]$.

The imbalance of excitatory and inhibitory neurotransmitters, lowered threshold of cortical excitability and impaired mechanisms for terminating electrical seizure activity is thought to predispose to these seizures. The seizure types include partial, generalized, simple partial with secondary generalization and generalized convul- 
sive status epilepticus [13].

Seizures in HIV-infected patients have a high rate of recurrence [14], thus initiation of antiepileptic therapy even after the first seizure is recommended [15]. Knowledge of the pharmacokinetic profiles of antiretroviral and antiepileptic drugs is essential as both groups of drugs are metabolized by the cytochrome P450 system. Fluctuations in serum antiepileptic and antiretroviral levels with loss of viral suppression have been found to occur with these combinations. The older generation AEDs lower the effective dose of non nucleotide reverse transcriptase inhibitors (NNRTIs) and protease inhibitors (PIs) with resultant virological failure and development of ARV resistance $[16,17]$. The newer antiepileptic agents like gabapentin, lamotrigine and levetiracetam do not have most of these disadvantages and are therefore recommended for use in HIV-associated seizures [18]. Birbeck et al. [19] reported that over $50 \%$ of patients with HIV potentially require therapy with antiepileptic drugs for the controls of seizures, psychiatric conditions or peripheral neuropathy and thus proposed guidelines for antiepileptic drug selection for people with HIV/ AIDS. The dearth of knowledge on the prevalence of seizures in Nigeria, Sub-Saharan Africa where HIV infection is endemically prompted this study.

\section{Methodology}

\subsection{Study Setting and Design}

The study was conducted at the HIV clinic of the Lagos State University Teaching Hospital, (LASUTH) one of the reference tertiary hospitals for HIV/AIDS in Lagos, Nigeria attending to about 8000 patients annually. We used a cross-sectional study design involving HIV positive adults, aged 18 years and above and on antiretroviral therapy (ART). Cases with a family history of epilepsy, previously diagnosed epilepsy, past history of febrile seizures, head injury, drug usage (IV heroin, marijuana) and metabolic derangements were excluded from the study. The institutional ethics review board approved the study and consent was obtained from the individual patients.

\subsection{Participant Recruitment and Data Collection}

A total of 308 HIV-positive cases were reviewed consecutively over a 1 year period between September 2011 and September 2012. Those with a first seizure during this 1 -year period were further examined by the first author (O.O.Os). Information documented include demographic data, the first seizure date, seizure characteristics, (frequency and clinical seizure type based on the 2010 International league against Epilepsy classification) and neurologic complications related to HIV at time of sei- zure. The CD4 cell count at the time of the first seizure was also documented from the database. This was followed by a detailed clinical examination of the nervous system. Seizure types were documented as focal, generalized, and focal with secondary generalization or status epilepticus.

\subsection{Data Analysis}

Data entry and analysis were achieved using the Statistical Package for Social Sciences (SPSS) version 19.0. Group differences in mean values of numerical data were compared using Students $t$ test, while Pearson chi-square was used to determine statistical significance of group differences for categorical variables.

Level of significance was set at $\mathrm{P}$ value $<0.05$.

\section{Results}

\subsection{Demographic Characteristics of Population with New-Onset Seizures}

During the study period, a total of 308 HIV-infected patients were reviewed in the out-patients department. Twenty $(20 / 308)$ had seizures in the last year. They were $6 / 20$ $(30 \%)$ males and $14 / 20(70 \%)$ females. Their ages ranged between $22-51$ years with a mean of $34.2 \pm 8.7$ years (median 34, range 29). The ages of the males ranged between $24-51$ years with a mean of $35.1 \pm 9.7$ years (median 32.5 , range 27) whilst that of the females ranged between $22-49$ years with a mean of $33.9 \pm 8.5$ years (median 34.5, range 27). The duration of cases on HAART ranged between $1-49$ months with a mean of 19.5 \pm 12.7 months (median 18.5, range 48) (Table 1).

\subsection{Frequency and Clinical Characteristics of Population with New-Onset Seizures}

Of the 308 participants, 20 (6.5\%) had new-onset seizures. The first seizure was focal in 2/20 (10\%) of cases whilst generalized seizures made up $90 \%(18 / 20)$ of cases. All generalized seizures were tonic-clonic whilst the focal seizures were without impairment of consciousness and with motor components only. Focal seizures with impairment of consciousness, focal seizures with secondary generalization and status epilepticus were not observed.

Table 1. Demographic characteristics of the study population.

\begin{tabular}{|c|c|}
\hline Variable & $\mathbf{N}=\mathbf{3 0 8}$ \\
\hline Age-range (years) & $22-51$ \\
\hline Mean age \pm SD (years) & $34.2 \pm 8.7$ \\
\hline Male:female ratio & $1: 2.3$ \\
\hline Mean duration on HAART (months) & $19.5 \pm 12.7$ \\
\hline Mean CD4 count (cells/mm³) & $165.3 \pm 145.7$ \\
\hline Frequency of new-onset seizures (\%) & $20(6.5)$ \\
\hline
\end{tabular}


At the time of first seizure, none of the cases had focal neurological deficit and the CD4 counts ranged between $30-518$ cells $/ \mathrm{mm}^{3}$ with a mean of $165.3 \pm 145.7(\mathrm{Me}-$ dian 124.0, range 488). Cases with CD4 counts of 200 cells $/ \mathrm{mm}^{3}$ and below constituted 70\% (14/20) whilst those with CD4 counts above 200 made up 30\% (6/20). $[\mathrm{p}=0.666 ; \mathrm{df}=5]$ A total of $13 / 20(65 \%)$ had recurrence of seizures whilst $7 / 20(35 \%)$ had single seizures during the study period. None of the patients had electroencephalogram nor imaging studies done as a result of financial constraints, as such, no cause was established for the seizures.

\section{Discussion}

New-onset seizures are not common in our HIV infected population $(6.5 \%)$. Wong et al. [20] had observed a higher rate of $11 \%$. Compared with the Wong study, the lower rate in our study may be attributed to the exclusion of cases with metabolic abnormalities from the onset. Pascual-sedano et al. had earlier reported that toxicmetabolic factors accounted for more than half (58.8\%) of the seizures patients experienced in their study [7]. Metabolic abnormalities are known to be a cause of seizures in HIV/AIDS and has also been found to increase the risk of status epilepticus. Also, in our study we included only HIV cases in the out-patients department. Exclusion of cases admitted in the emergency medical wards may account for the difference. Van Paesschen et al. [3] and Pascual-sedano et al. [7] however reported lower rates of $4 \%$ and $3 \%$ respectively.

We report a higher number of cases $(90 \%)$ with generalised seizures. As in our study, other authors had reported generalised seizures to be more common than focal seizures. Wong et al., Pascual-Sedano et al. and Bartholomei et al. had reported $94 \%, 70.6 \%$ and $70 \%$ cases of generalized seizures in their series respectively $[7,13,20]$. We did not observe any case of status epilepticus in our series probably because of our inclusion criteria. We studied only cases in the out-patients clinics.

Also, the exclusion of cases with metabolic abnormalities which is known to increase the risk for status epilepticus may also account for this. Pascual-sedano et al. had reported $17.6 \%$ of cases of status epilepticus in a cohort of in-patients.

Fourteen $(70 \%)$ cases in our study had severe immune suppression as evidenced by low CD4 cell counts, at the time of their first seizure. This finding is similar to the $55.7 \%-91.0 \%$ reported in previous studies [2,3,13,21]. Dore et al. [4] in a case-control study observed that $84 \%$ of patients with new-onset seizures had prior AIDSdefining illness with a low mean CD4 T-cell count. Majority of seizures are known to occur in the advanced stages of HIV infection and their apparent implications may be explained through HIV opportunistic CNS pa- thologies [6].

Seizures were reported as the presenting manifestation of HIV-related disease in $3.8 \%$ to $18 \%$ of patients in some studies $[2,13,20]$. We did not observe seizures as the initial manifestation of HIV infection in our population, just as Pascual-sedano et al. [7] had also reported. We were unable to identify the cause of seizures in our study as imaging studies were not done as a result of financial constraints.

None of our cases presented with focal neurological deficit at the time of their first seizure, even with an overall low mean CD4 cell count of 165 cells $/ \mathrm{mm}^{3}$ and a mean duration of 19.5 months on highly active anti-retroviral therapy. The absence of focal signs which is a pointer of underlying mass lesions and the preponderance of generalised seizures which suggests a more diffuse brain disease may support HIV encephalopathy as the main cause of seizures in our population. Previous literature had documented that approximately half of HIVinfected patients with seizures have no definite identifiable disease of the brain, and cerebral HIV infection seems to be the most likely cause of the seizures $[2,4,7,20]$.

\section{Conclusion}

Seizures remain infrequent neurological manifestations of HIV patients on HAART with a high recurrence rate. Most occur with severe immune suppression and may be attributable to HIV encephalopathy. Early treatment with anti-epileptic drugs would reduce this burden and improve the quality of life in these patients.

\section{Limitations}

Limitations of our study include lack of neuroimaging studies such as computed tomography (CT) and magnetic resonance imaging (MRI) as a result of financial constraints. This hampered aetiological diagnosis thus limiting diagnostic evaluations.

\section{REFERENCES}

[1] C. Kellinghaus, C. Engbring, S. Kovac, G. Möddel, F. Boesebeck, M. Fischera, et al., "Frequency of Seizures and Epilepsy in Neurological HIV-Infected Patients," Seizure: European Journal of Epilepsy, Vol. 17, No. 1, 2008, pp. 27-33. doi:10.1016/j.seizure.2007.05.017

[2] D. Holtzman, D. Kaku and Y. So, "New-Onset Seizures Associated with HIV Infection: Causation and Clinical Features in 100 Cases," American Journal of Medicine, Vol. 87, No. 2, 1989, pp. 173-177. doi:10.1016/S0002-9343(89)80693-X

[3] W. Van Paesschen, C. Bodian and H. Maker, "Metabolic Abnormalities and New-Onset Seizures in Human Immunodeficiency Virus-Seropositive Patients," Epilepsia, Vol. 36, No. 2, 1995, pp. 146-150. doi:10.1111/j.1528-1157.1995.tb00973.x 
[4] G. J. Dore, M. G. Law and B. J. Brew, "Prospective Analysis of Seizures Occurring in Human Immunodeficiency Virus Type-1 Infection," Journal of Neuro-AIDS, Vol. 1, No. 4, 1996, pp. 59-69. doi:10.1300/J128v01n04 06

[5] B. Navia, B. Jordan and R. Price, "The AIDS Dementia Complex: I Clinical Features," Annals of Neurology, Vol. 19, No. 6, 1986, pp. 517-524. doi:10.1002/ana.410190602

[6] M. Modi, A. Mochan and G. Modi, "New Onset Seizures in HIV-Seizure Semiology, CD4 Counts, and Viral Loads," Epilepsia, Vol. 50, No. 5, 2009, pp. 1266-1269. doi:10.1111/j.1528-1167.2008.01942.x

[7] B. Pascual-Sedano, A. Iranzo, J. Marti-Fabregas, et al., "Prospective Study of New-Onset Seizures in Patients with HIV Infection: Etiologic and Clinical Aspects," Archives of Neurology, Vol. 56, No. 5, 1999, pp. 609-612. doi:10.1001/archneur.56.5.609

[8] J. M. Provenzale and J. R. Jinkins, "Brain and Spine Imaging Findings in AIDS Patients," Radiologic Clinics of North America, Vol. 35, 1997, pp. 1127-1166.

[9] D. J. Farrar, T. P. Flanigan, N. M. Gordon, R. L. Gold and J. D. Rich, "Tuberculous Brain Abscess in a Patient with HIV Infection: Case Report and Review," American Journal of Medicine, Vol. 102, No. 3, 1997, pp. 297-301. doi:10.1016/S0002-9343(97)00386-0

[10] C. Martinez-Vazquez, J. Bordon, A. Rodriguez-Gonzalez, et al., "Cerebral Tuberculoma-Acomparative Study in Patients with or without HIV Infection," Infection, Vol. 23, No. 3, 1995, pp. 49-53. doi:10.1007/BF01793855

[11] A. N. Mamelak, W. G. Obana, J. F. Flaherty and M. L. Rosenblum, "Nocardial Brain Abscess: Treatment Strategies and Factors Influencing the Outcome," Neurosurgery, Vol. 35, No. 4, 1994, pp. 622-631. doi:10.1227/00006123-199410000-00007

[12] D. R. Labar, "Seizures and HIV Infection," In: T. A. Pedley and B. S. Meldrum, Eds., Recent Advances in Epilepsy, Churchill Livingstone, Edinburgh, 1992, pp. 119126.

[13] F. Bartolomei, M. Gavaret, C. Dhiver, et al., "Isolated, Chronic, Epilepsia Partialis Continua in an HIV-Infected Patient," Archives of Neurology, Vol. 56, No. 1, 1999, pp. 111-114. doi:10.1001/archneur.56.1.111
[14] D. S. Chadha, A. Handa, S. K. Sharma, P. Varadarajulu and A. P. Singh, "Seizures in Patients with Human Immunodeficiency Virus Infection," Journal of the Association of Physicians, Vol. 48, 2000, pp. 573-576.

[15] P. Mullin, G. Green and R. Bakshi, "Special Populations: the Management of Seizures in HIV-Positive Patients," Current Neurology and Neuroscience Reports, Vol. 4, No. 4, 2004, pp. 308-314. doi:10.1007/s11910-004-0057-x

[16] P. W. Hugen, D. M. Burger, K. Brinkman, H. J. ter Hofstede, R. Schuurman, P. P. Koopmans, et al., "Carbamazepine-Indinavir Interaction Causes Antiretroviral Therapy Failure," Annals of Pharmacotherapy, Vol. 34, No. 4, 2000, pp. 465-470. doi:10.1345/aph.19211

[17] M. Barry, S. Gibbons, D. Back and F. Mulcahy, "Protease Inhibitors in Patients with HIV Disease. Clinically Important Pharmacokinetic Considerations," Clinical Pharmacokinetics, Vol. 32, No. 3, 1997 pp. 194-209. doi:10.2165/00003088-199732030-00003

[18] M. D. Liedtke, S. M. Lockhart and R. C. Rathbun, “Anticonvulsant and Antiretroviral Interactions," Annals of Pharmacotherapy, Vol. 38, No. 3, 2004, pp. 482-489. doi:10.1345/aph.1D309

[19] G. L. Birbeck, J. A. French, E. Peruca, D. M. Simpson, H. Fraimow, J. M. George, et al., "Evidence-Based Guideline: Antiepileptic Drug Selection for People with HIV/ AIDS. Report of the Quality Standards Subcommittee of the American Academy of Neurology and the Ad Hoc Task Force of the Commission on Therapeutic Strategies of the International League Against Epilepsy," Neurology, Vol. 78, No. 2, 2012, pp. 139-145.

[20] M. C. Wong, N. D. A. Suite and D. R. Labar, "Seizures in Human Immunodeficiency Virus Infection," Archives of Neurology, Vol. 47, No. 6, 1990, pp. 640-642. doi:10.1001/archneur.1990.00530060048015

[21] A. Millogo, D. Lankoandé, I. Yaméogo, A. A. Yaméogo, A. Sawadogo and A. B. Sawadogo, "New-Onset Seizures in Patients with Immunodeficiency Virus Infection in Bobo-Dioulasso Hospital (Burkina Faso)," Bulletin de la Société de Pathologie Exotique, Vol. 97, No. 4, 2004, pp. 268-270. 